\title{
Distributed Database Access Technology Research Based on the .Net
}

\author{
HE Dinghua, a \\ ${ }^{1}$ Department of Computer, Wuhan Polytechnic, Wuhan 430074, China \\ ahedinghuawh@126.com
}

Keywords: .NET Framework; Database Technique; Distributed System; Could Computing.

\begin{abstract}
For big data analysis and processing of the SQL database has the very good read and write performance and scalability, but cannot support a complete SQL queries and inter-bank transactions across the table, which is mainly composed of a relational database for traditional financial business have limits on the application. The OceanBase is facing huge amounts of data query of distributed database, combines the advantages of relational database and relational database, support relational query and an inter-bank transactions across the table at the same time, the extensible. However, at present OceanBase supports only simple, the nested children the SQL query which can't very well to support the application. Therefore, we design a novel system to overcome the drawbacks in this paper. The experiment shows the feasibility of our approach.
\end{abstract}

\section{Introduction}

With the further development of cloud computing and web technology, no database is growing stronger. No database to abandon the traditional relational database strictly transactional consistency and paradigm of constraints, the weak consistency model, support distributed and horizontal extension, meet the needs of a lot of data management. To information security and reduce the database system to upgrade maintenance, domestic Banks began to advances the strategy of "go to the IOE". No data than traditional relational database which is ultra-high cost performance and good scalability. These qualities make no database be the first choice of the domestic banking sector deal with huge amounts of data. Database OceanBase is Alibaba group developed a massive distributed relational database system, adopted the no database architecture, based on the lateral extension mode, can be adjusted by means of dynamic increase/decrease online server system load which has a good scalability. Moreover, the system realized the important characteristics of a relational database, the SQL query language support, relative to other no (database, to better meet the needs of the financial business. OceanBase scalability, strong standard SQL query and transaction consistent sexual function, in response to the banking financial business, has a big advantage [1-3]. A financial business is characterized by the extensive use of nested children query, but for now, OceanBase supports only simple nested SQL, for complex query embedded within the cover of unrealized, thus hinders the financial business of import. Cloud computing environment, in response to the challenges brought about by the huge amounts of data and user request, to solve the large-scale data access bottleneck problem facing the traditional database, distributed cache technology to introduce, to provide users with high performance, high availability, scalable data cache service.

Distributed cache the data distribution to multiple cache service node data in memory management, provides a unified access interface, based on redundancy backup mechanism to achieve high availability support, also known as memory data grid. Compared with the relational database technology, however, because of the lack of relatively uniform data model and standardization of data access technology, in many sources, heterogeneous, distributed spatial data sharing and integration and interoperability lags far behind in terms of relational database technology, restricts the further develop the technology and application of geographic information [4]. At present, the field of spatial information has more than 100 kinds of commonly used spatial data format and integrated information system of the application of various departments as demand differences and historical reasons tend to adopt different vendor's format data, communication between departments (Internet visits) become a thorny problem. Heterogeneous, multi-source spatial data sharing and 
interoperability technology solutions, data format conversion model, direct data access mode, relational database space expansion mode, and data integration technology of Web Service. These methods to a certain extent solve the problem of the spatial data sharing and interoperability, but there are also some limitations. The figure 1 shows the situation.

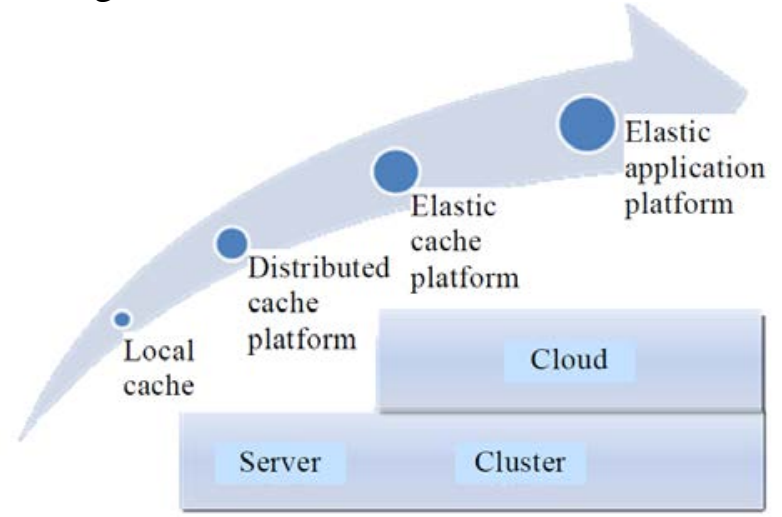

Fig. 1The Structure and Development of Distributed Cache

To overcome the mentioned drawbacks and difficulties, we conduct research on distributed database access technology in this paper. The detailed research will be introduced in the following.

\section{Technique Description and Corresponding Organization}

With the rapid development of computer network technology, more and more network information, database access technology is our concern all the people more and more, ADO. Net data access technology has gained wide support and praise. In the past, most Web pages are static letter pastor, composition, the site only allows visitors to read data, site interaction is not strong, and the information is not stored visitor, if you must store the visitor information, you need to use the database and data access strategy, allowing data access strategy, allowing the programmer and database connection, and to provide search, insert, update and delete data command. ADO. Net effective data manipulation, data access is decomposed into multiple can be used alone. ADO. Net SQL Server, OLEDB and XML data source access, user applications can use the ADO. It is easy to connect to the data source, data retrieval and update operations [5]. The network structure as shown in figure 2 below.

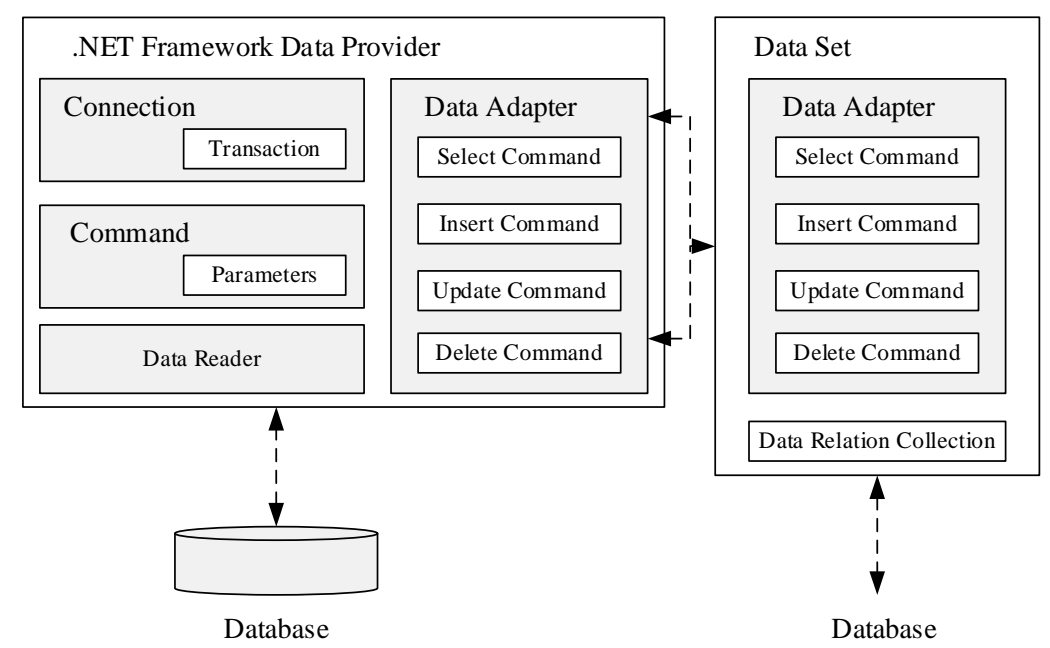

Figure 2. System structure of ADO.Net

Model and Structure of the System. There are two most common ways of system data management: using file system data and make use of the database system management data. The former can satisfy the requirement of system real time, but to write the data type of the file system should be according to the project need to define, a large number of programming jobs are spent on the data organization, break up, make whole programming work greatly increased, and the file 
number is likely to lead to chaos in the management. Existing measurement task is generally adopted the latter to store data for management and use. The formula 1 shows the cluster structure.

$$
C_{m \times i}=\left[\begin{array}{cccc}
d n_{11} & d n_{12} & \cdots & d n_{1 i} \\
d n_{21} & d n_{22} & \cdots & d n_{2 i} \\
\vdots & \vdots & & \vdots \\
d n_{m 1} & d n_{m 2} & \cdots & d n_{m i}
\end{array}\right]
$$

Powerful database function such as data storage, query, calls for industrial automation and testing and measuring system with a powerful technical support, the user can be used to create a database to manage complex testing tasks, storage, test data and can summarize the test result of automatic test system. The figure 3 shows the organization.

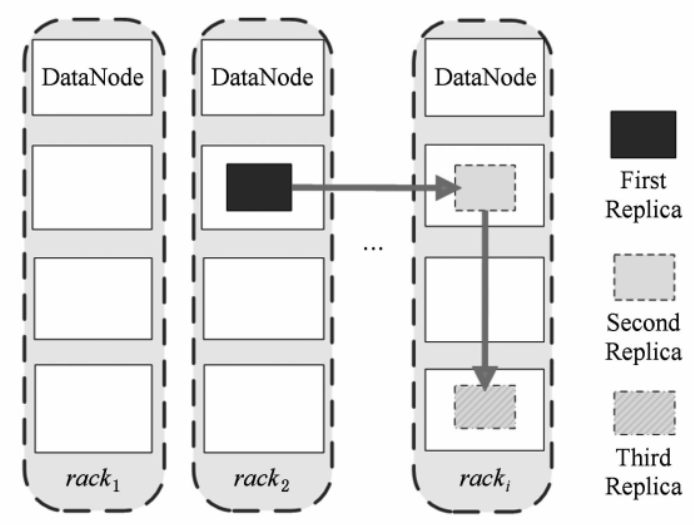

Fig. 3 The Structure of the System Organization

Dynamic extension online add or remove nodes to achieve the adjustment of the cache capacity, at the same time support services available. Capacity planning to provide support for elastic supply, its goal is to determine the initial and the adjusted cache capacity and number of nodes. Due to the size of the data access and model with time-varying, lead to some cache node and become a bottleneck restricting the throughput of the whole system. Elimination of load balance board on the system's performance and service quality assurance plays an important role. Such as load balance goal is to make the cache data and access load uniformly distributed, as far as possible between the nodes contain client equilibrium and the service side equilibrium two implementation methods.

System Working Mechanism. Data migration is the key to realize the dynamic extension of nodes and load balance technology. At present, most of the distributed cache support online data migration, but a lack of balance of hot data migration processing. It should be pointed out that, in the process of data migration with a large number of state synchronization can bring some impact to the system performance, therefore, how to effectively reduce the migration overhead is the need to address the problem. The figure 4 shows the flowchart.

Cloud computing has the characteristics of open, dynamic and changeable these characteristics determine the distributed cache needs to be agile, initiative, and adaptability. Described the system agility and adaptability in accordance with the change of internal and external condition, such as the request data quantity and proportion of reading and writing, etc. to respond in a timely manner and the ability to adjust, the dimension is closely associated with elastic supply dimensions. In-depth analysis of the characteristics of different caching strategies and the application of scene is conducted which is of great significance to the implementation of the adaptive mechanism. According to different strategy target, the caching policy progress can be divided into several dimensions, such as topology, cache replacement algorithm and data consistency strategy, etc. Near district strategy based on partitioning strategies to increase the capacity of a smaller front-end cache, is used to accelerate the hot data access [6-7]. User request object, can be no delay in the front-end cache loading, won't be loaded automatically from the back-end cache loading. The back-end cache capacity is larger but not as good as the front-end cache access speed. Replication technology's main goal is to improve the 
availability, at the same time can be balanced by creating a copy of the data node load and improve the system performance. From the perspective of replication model replication techniques can be divided into master-slave replication and more master replicates. Master-slave replication, read and write operations are executed in the master copy, to update master copy is forwarded to this; Master replicates, allowing any copy executed more read and write operations, forward to other copies. Each copy will update replication technology from the time dimension and can be divided into synchronous replication and asynchronous replication. Synchronous replication, the client needs to wait for a copy operation is complete to get response, performance overhead, but can guarantee the instant a sex; Asynchronous replication without waiting for the copy operation completed can return a result, has a better performance and scalability, and can guarantee the data consistency, eventually but at the expense of the instant consistency, this means that applications may read at a certain moment to inconsistent data. The experiment will be conducted in the next section.

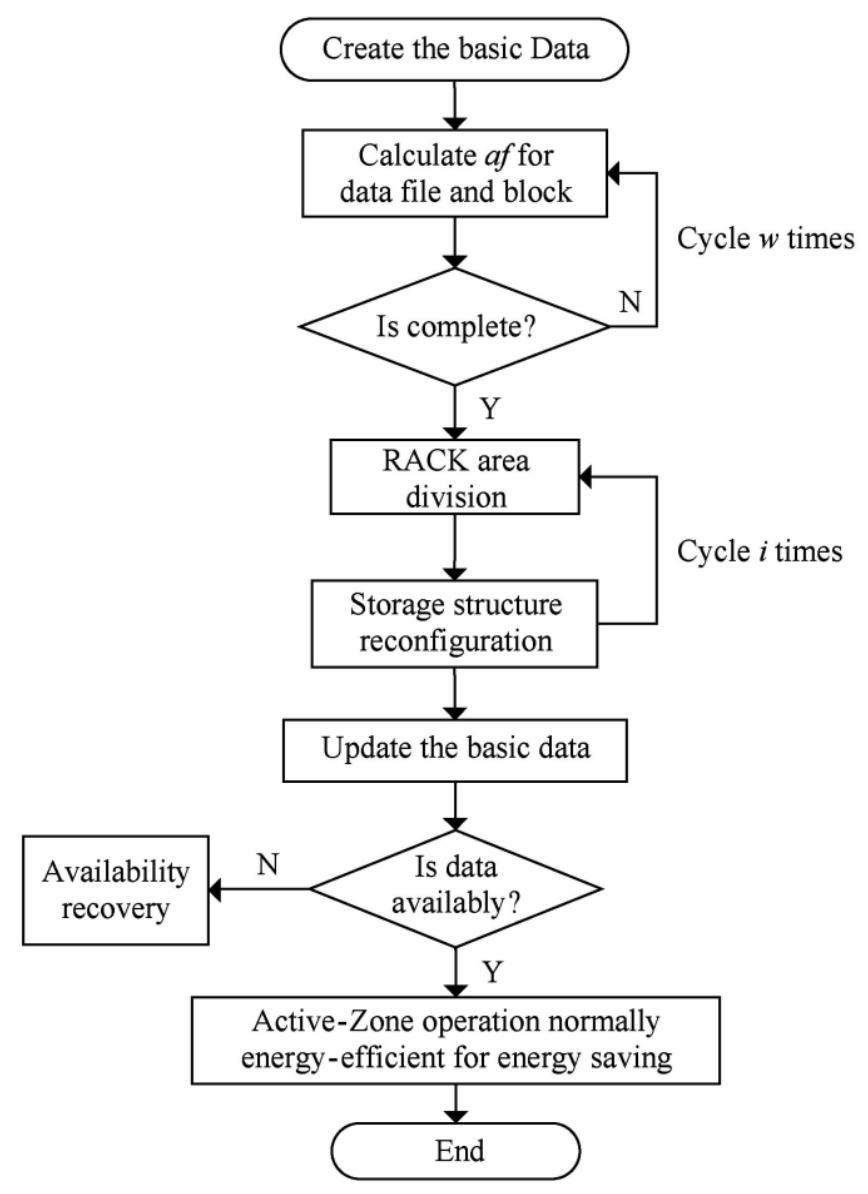

Fig. 4 The Flowchart and Logistics of the System

\section{Experiment and Result}

In order to validate the proposed method, we conduct comparison experiment in the section. The experiment to measure mass subquery data set the performance of the nested children query strategy under the condition of same experimental environment. Under the large-scale subquery data set of nested query strategy, performance test results and performance of the testing is shown below figure 5. 

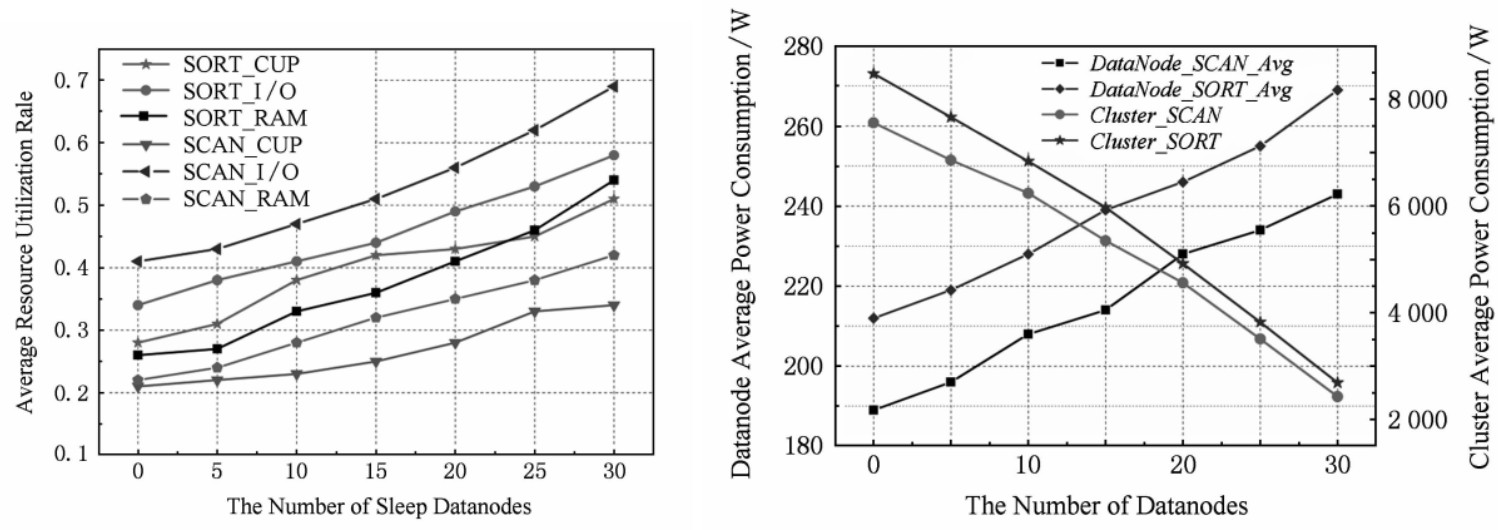

Fig. 5 The Result of the Comparison Experiment

\section{Conclusion and Summary}

The introduction of cloud computing, effectively promote the profound changes in the field of IT, at the same time also to the development of distributed cache technology has brought rare opportunities. As the cloud platform promotion application performance is an important means of distributed cache technology in recent years has received the extensive concern of the industry and academia. To level up the current methodology, we conduct research on distributed database access technology in this paper. The experiment result proves the correctness of the proposed system.

\section{Acknowledgements}

Scores query based on Android mobile App design and development, Subject of Wuhan Polytechnic, 2014.

\section{References}

[1] N Z, Gao Y. Research on the Implementation Methods of Security Management of Distributed Database Application System[J]. Machine Tool Technology Mechatronics \& Information Engineering, 2014.

[2] Thanh Tan, Le . Channel Assignment With Access Contention Resolution for Cognitive Radio Networks[J]. Vehicular Technology, IEEE Transactions on, 2014, 61(6):2808 - 2823.

[3] Hagsten E. Distributed access to linked microdata: the example of ICT and exports[J]. Applied Economics Letters, 2014.

[4] Cao H, Leung V, Chow C, et al. Enabling technologies for wireless body area networks: A survey and outlook[J]. Communications Magazine, IEEE, 2009, 47(12): 84-93.

[5] Zhang Y, Yu R, Nekovee M, et al. Cognitive machine-to-machine communications: visions and potentials for the smart grid[J]. Network, IEEE, 2012, 26(3): 6-13.

[6] Raouf A E A, Badr N L, Tolba M F. Dynamic Distributed Database over Cloud Environment[J]. Communications in Computer \& Information Science, 2014.

[7] Zhang Q, Cheng L, Boutaba R. Cloud computing: state-of-the-art and research challenges[J]. Journal of internet services and applications, 2010, 1(1): 7-18. 